\title{
Stabilization of soy milk using konjac glucomannan
}

\author{
Kai An ${ }^{1}$, Huiting Kang ${ }^{1}$, Dating Tian ${ }^{1,2 *}$ \\ ${ }^{1}$ School of Chemical and Environmental Engineering, Hubei Minzu University, Enshi 445000, People's Republic of China, ${ }^{2}$ Key Laboratory of \\ Biologic Resources Protection and Utilization of Hubei Province, Hubei Minzu University, Enshi 445000, People's Republic of China
}

\section{A B S T R A C T}

A novel compound functional plant beverage could be formed by blending soy milk with konjac glucomannan (KGM), a beneficial polysaccharide. Both the phase behavior and the stability of KGM-soy milk blend system were studied. The phase diagram showed that the KGM-soy milk blend system could form three regions: i.e., Unstable zone, metastable zone and stable zone. The stability of the blend system was investigated by measuring turbidity, viscosity, Zeta potential, particle size and differential scanning calorimetry (DSC), respectively. The microstructure of the KGM-soy milk mixed system was characterized by confocal laser scanning microscope (CLSM), which indicated that KGM and soy milk could be evenly dispersed under certain conditions. The mixtures with relatively low KGM component were unstable and easy to sedimentation. However, the blend systems with high KGM component were relatively stable and were not easy to occur phase separation. The results implied that KGM could act not only as a functional ingredient, but also as a stabilizer in the compound plant beverage.

Keywords: Konjac glucomannan; Soy milk; Phase behavior; Stability; Beverage

\section{INTRODUCTION}

Konjac glucomannan (KGM) is a type of natural polysaccharide extracted from the tuber of Konjac, which composed of D-glucose and D-mannose linked by $\beta-1,4$ glycosidic bond and the ratio of the two substances is approximately 1:1.6. KGM possesses good water solubility, thickening, gelation and film formation. The unique physical and chemical properties of KGM make it can be widely used in many fields including food industry. In Japan, as a traditional healthy food, the industry of KGM has developed rapidly since 1860s and KGM has been approved as healthy food and food additive. The Food and Drug Administration (FDA) of the United States listed it as a food additive in 1997, and the European Union approved it as a food additive in 1998. At present, China has also approved it as a food additive and food raw material.

KGM can be made into konjac tofu, konjac noodles, konjac bread, konjac meat pieces and other foods. KGM foods have the characteristics of low fat, low calorie and high fiber, which not only have delicious and pleasant taste, but also have some functions such as lowering blood sugar, lowering blood pressure, lowering blood fat, reducing weight, preventing cancer and so on. Moreover, the addition of KGM in food not only endows some functions with food, but also improves the microstructure, texture, appearance and shelf life of food(Behera and Ray, 2016).

As it is well known, soy milk is a traditional vegetable protein beverage in Asia, especially in China, which is made from soybean by soaking, grinding and filtering. Soy milk not only is rich in protein, fat and carbohydrate, but also contains calcium, phosphorus, iron and a variety of vitamins(Giri and Mangaraj, 2012; Ahmed et al., 2019). In addition, soy milk also contains soybean saponins, isoflavones, lecithin and etc., which have potential effects of lowering cholesterol, hepatoprotection, anti-cancer, anti-oxidation and antihuman-immunodeficiency-virus(Omoni and Aluko, 2005). Soy milk also has a high absorption rate of the human body, and therefore, it especially is favored by vegetarians and lactose intolerant persons.

However, protein beverage is prone to sedimentation during storage, which affects its taste and quality. In order to overcome the phase separation of protein beverage, people usually add certain amount of polysaccharide into a beverage, which utilize the interaction between polysaccharide and

\footnotetext{
*Corresponding author:

Dating Tian, School of Chemical and Environmental Engineering, Hubei Minzu University, Enshi 445000, People's Republic of China. E-mail: tiandating@163.com
}

Received: 14 February 2019; Accepted: 17 May 2019 
protein to improve the stability of the system(Abbasi and Mohammadi, 2013; Zhao et al., 2013; Chun et al., 2014; Dai et al., 2017). The addition of natural polysaccharides as health-supporting nutrients to dairy products has been carried out for many years, however, the thermodynamic incompatibility between proteins-polysaccharides might affect the stability of polysaccharide-added dairy products (Turgeon et al., 2003). It is generally believed that under the combined influence of entropy and enthalpy, two different mechanisms of phase separation of the blending system can be observed(de Kruif and Tuinier, 2001). Namely, if there is a net repulsion between two biopolymers, the mixture segregates due to the mutual repulsion, and if two biopolymers attract one another, phase separation may also occur for the association. Currently, the research of using polysaccharides to improve protein beverages has been attracted increasing attention. The microstructure, appearance and quality of the protein beverages could be improved by using pectin as a stabilizer, reported by Boulenguer's group (Laurent and Boulenguer, 2003) and Yuliarti's group(Yuliarti et al., 2019). Hussein et al (Hussein et al., 2011) extracted six plant polysaccharides from okra fruits, whole plant Jew's-mallow and taro corn, and successfully used these products to improve the texture, body, appearance and flavor of yoghurt. Recently, Corke's group(Dai et al., 2017) reported the research of using KGM as potential health-supporting nutrient to improve the quality of fresh cow's milk.

As far as we know, there is no literature has yet been reported on the influence of the KGM addition to fresh soy milk. The reasons why KGM and soy milk are used to prepare the compound beverage are as follows. Firstly, adding a proper amount of KGM in soy milk could combine the advantages of plant protein and plant polysaccharide, and strengthen the functions of the beverage in preventing obesity, hyperlipidemia, hypertension and diabetes. Secondly, since two raw materials all come from plants, which do not contain lactose in animal dairy products, the products obtained would be especially suitable for people who are lactose intolerant. Thirdly, the compound beverage obtained in this work is zero additive, which is safe for human body. Moreover, as a polysaccharide, KGM might improve the stability of protein beverage.

Therefore, in this paper, KGM was blended with fresh soy milk to prepare a novel functional compound plant beverage. Then, various methods such as turbidity, viscosity, Zeta potential, particle size, DSC and CLSM were used to investigate the stability, phase behavior, thermal property and microstructure of KGM-soy milk mixture, which might provide basic data for the development of this new compound functional plant beverage.

\section{MATERIALS AND METHODS}

\section{Materials}

Fresh soy milk was made in our lab by soy milk maker and soybean dregs were removed before use, which contained water $(88.7 \%, \mathrm{w} / \mathrm{w})$, proteins $(3.8 \%, \mathrm{w} / \mathrm{w})$, fat $(3.5 \%, \mathrm{w} / \mathrm{w})$ and other components $(4.0 \%, \mathrm{w} / \mathrm{w})$. KGM powder was purchased from Hubei Yizhi Konjac Biotechnology Co., Ltd, and refined with ethanol and dried under vacuum condition before use.

\section{Preparation of the KGM-soy milk blend system}

A certain amount of KGM powder was dissolved in distilled water, and stirred for $2 \mathrm{~h}$ to make a series of KGM solutions with concentrations ranged from $0.1 \%$ to $2.5 \%(\mathrm{w} / \mathrm{v})$. Stirred fully for $2 \mathrm{~h}$ at room temperature, $10 \mathrm{~mL}$ of KGMsoy milk mixture was prepared by blending KGM solution with fresh soy milk in different proportions (for example, at the volume percentage of soy milk from $0 \%$ to $100 \%$ ).

\section{Phase separation diagram}

KGM-soy milk mixture was poured into the glass centrifugal tube, standing for $12 \mathrm{~h}$ under $5^{\circ} \mathrm{C}$. The phase separation phenomenon of the mixture was observed visually and then the phase separation diagram was plotted.

\section{Apparent viscosity}

In this work, the apparent viscosity of different KGMsoybean milk mixture with $60 \%(\mathrm{v} / \mathrm{v})$ volume percentage of soybean milk was measured by the rotating viscosimeter (NDJ-4, Shanghai Precision Instrument Company, China) (Sharafbafi et al., 2014).

\section{Turbidity}

$0.2 \mathrm{~mL}$ of the KGM-soy milk was diluted 200-fold with distilled water, and the turbidity of the mixture was measured in the colorimetric cup using a photoelectric turbidimeter (WGZ-200, Shanghai Precision Instrument Company, China).

\section{Zeta potential}

At room temperature, $0.2 \mathrm{~mL}$ of the KGM-soy milk mixture was diluted 500-fold with distilled water, and then was slowly injected into the Zeta potential sample pool of the Zetasizer(Nano-ZS, Malvern Instruments, $\mathrm{UK})$ to measure the Zeta potential in the absence of bubbles(Abbasi and Mohammadi, 2013).

\section{Particle size distribution}

At room temperature, $0.2 \mathrm{~mL}$ of KGM-soy milk mixture was diluted 500 -fold with distilled water, and the particle size was measured in polystyrene colorimeter by the dynamic light scattering (Nano-ZS, Malvern Instruments, UK)(Ni et al., 2018). 


\section{Differential scanning calorimetry}

The thermal behavior of samples was investigated by differential scanning calorimeter (DSC 404F3, Netzsch, Germany). The temperature rose from $25^{\circ} \mathrm{C}$ to $250^{\circ} \mathrm{C}$ at a heating rate of $5^{\circ} \mathrm{C} / \mathrm{min}$, and the purging rate of nitrogen was $40 \mathrm{~mL} / \mathrm{min}$ accompanied by the purging rate of shielding gas of $20 \mathrm{~mL} / \mathrm{min}$ (Pirestani et al., 2018).

\section{Laser scanning confocal scanning microscope}

The laser scanning confocal scanning microscope was observed using a CLSM system (FV1200 Dichroic mirror DM570, Olympus Corporation, Tokyo, Japan), according to the literatures with some modification(Dai et al., 2017; Ni et al., 2018). Nile red was used as fluorescent dye and excitation wavelength was $534 \mathrm{~nm}$.

\section{RESULTS AND DISCUSSION}

\section{Phase behavior}

Fig. 1 shows visual images of the phase separation of KGM-soy milk mixture before standing(above) after standing for $12 \mathrm{~h}$ (below). The groups of (i), (ii) and (iii) illustrate the images of some typical mixture which phase separation easily occur, those of the mixtures with $60 \%$ of soy milk concentration and 0.1-2.5\% KGM concentration, and those of mixtures with $10-90 \%$ soy milk concentration and $0.5 \%$ of KGM concentration, respectively. According to the results of phase separation, the phase behavior diagram could be plotted (Fig. 2). The KGM- soy milk mixture system after stood for $12 \mathrm{~h}$ formed three typical systems: unstable system, metastable system, and stable system (Ni et al., 2018). KGM-soy milk mixture that freshly prepared all were homogeneous, however, with the increase of the static settlement time, the unstable mixture gradually sedimented. After $12 \mathrm{~h}$, flocculating suspensions were formed in the lower layer of the metastable mixture, and the upper layer was transparent but the phase interface was blurred. For the unstable system, there was a phase separation between the lower white precipitation layer and the upper transparent layer, where the interface between two phases was clear. Generally, when the amount of KGM was high, the mixture was in a homogeneous phase, and there was no significant difference before and after standing, i.e., Phase separation did not occur, which meant that it was a stable system.

Fig. 2 shows the phase diagram of the KGM-soybean milk mixture system after standing for $12 \mathrm{~h}$ at $5^{\circ} \mathrm{C}$. It could be found from the phase separation diagram that the stable phase area expanded with the increase of the KGM concentration. For the mixed system prepared at low concentration of KGM solution, for example, at $0.1-0.5 \%(\mathrm{w} / \mathrm{v})$, phase separation did not occur only in low percentage of soy milk. That might be attributed to that KGM molecule did not completely cover soy proteins, and colloidal particles agglomerated and settled due to the interaction of proteins. The KGM macromolecules were not able to completely cover proteins, and protein molecules could interact with each other, causing agglomeration of particle and sedimentation of protein. In addition, the phase separation diagram showed that

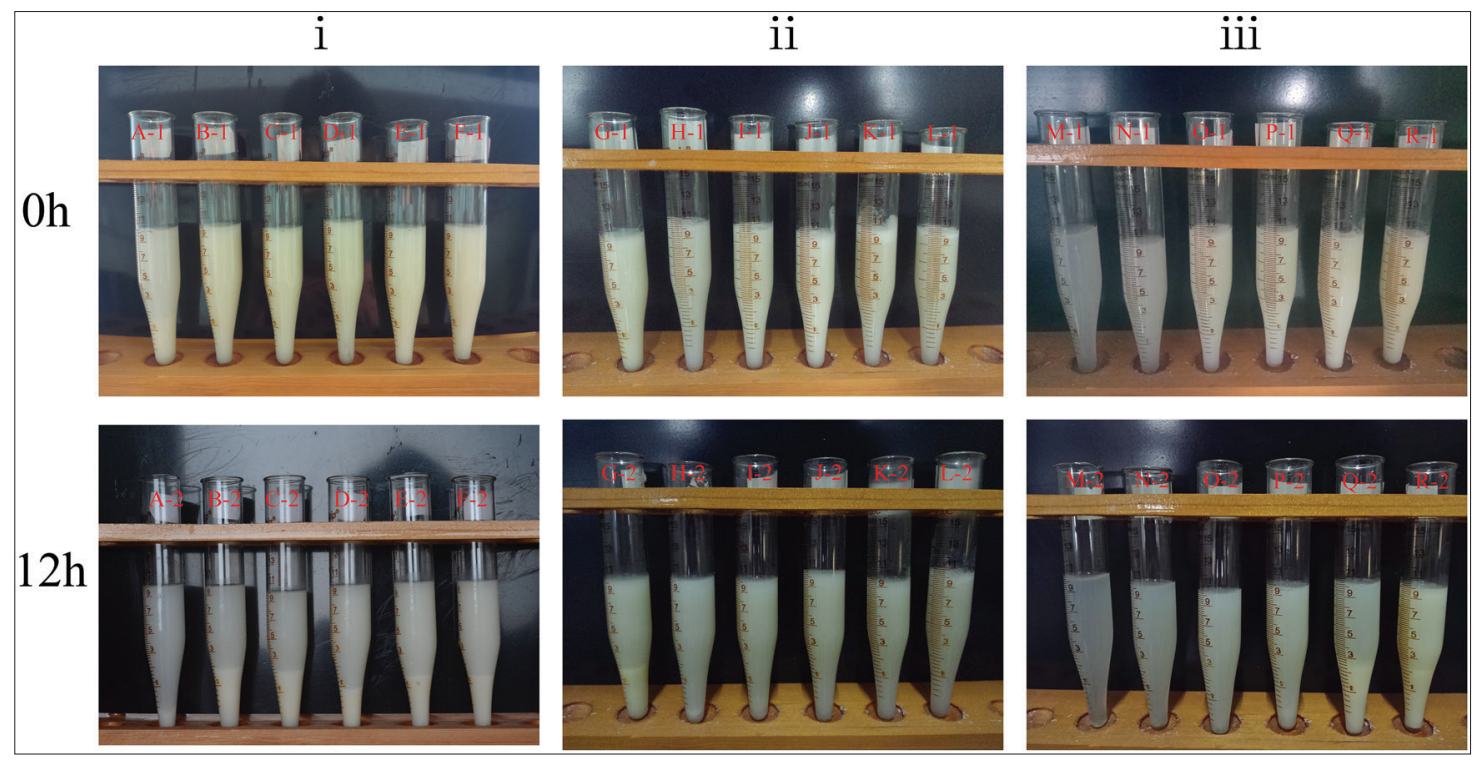

Fig 1. Visual images of KGM- soy milk mixture samples before standing(above) and after standing for $12 \mathrm{~h}\left(\right.$ below) at $5{ }^{\circ} \mathrm{C}(\mathrm{A}: 0.1 \% \mathrm{KGM}-40 \%$ soy milk; B: $0.5 \%$ KGM-80\% soymilk; C: $1.0 \%$ KGM-90\% soy milk; D: 1.5\% KGM-90\% soy milk; E: 2.0\% KGM-95\% soy milk; F: 2.5\% KGM-95\% soy milk; G: 0.1\% KGM-60\% soy milk; H: 0.5\% KGM-60\% soy milk; I: $1.0 \%$ KGM-60\% soy milk; J: $1.5 \%$ KGM-60\% soy milk; K: $2.0 \%$ KGM-60\% soy milk; L: $2.5 \%$ KGM-60\% soy milk; M: 0.5\% KGM-10\% soy milk; N: 0.5\% KGM-20\% soy milk; O: 0.5\% KGM-40\% soy milk; P: 0.5\% KGM-60\% soy milk; Q: $0.5 \%$ KGM- $80 \%$ soy milk; R: $0.5 \%$ KGM- $90 \%$ soy milk). 
phase separation occurred in almost all mixed systems containing more than $90 \%(\mathrm{v} / \mathrm{v})$ of soybean milk, indicating that when the concentration of KGM was low, the KGM molecules cannot completely cover protein particles, and then the sedimentation occurred. The stable area without phase separation in the phase diagram was homogeneous KGM-soy milk mixture system, revealing the compatibility of KGM- soybean milk under certain condition (Chun et al., 2014). For almost all mixtures with phase separation, when shaken again by hand, they were redistributed evenly, which might be the interaction between colloidal particles was relatively weak, and also meant that the phase separation of KGM-soy milk mixture was reversible(Ye and Harte, 2014).

The overall stability and microstructure of the KGM-soy milk mixed system were not only related to the physical and chemical properties of soy milk or KGM, but also related to the viscosity of the system(Xiong et al., 2018). The KGM-soy milk mixed system appeared to be "stable" under certain condition, but showed phase separation within a certain storage time. The rate of phase separation of mainly depended on the viscosity of the blended system(Kontogiorgos et al., 2009; Chun et al., 2014). The viscosity of the mixed system with high KGM concentration was higher, which made mixtures formed a network structure, preventing the aggregation of protein particles and contributing to form a stable system (Ni et al., 2018). The research of Dalgleish(Dalgleish, 2006) also showed that the colloidal properties of polysaccharides could stabilize the emulsion to prevent flocculation and accumulation. Meanwhile, adding colloid into the emulsion, the emulsion system could form compact and dense droplets, then, the emulsion showed good stability.

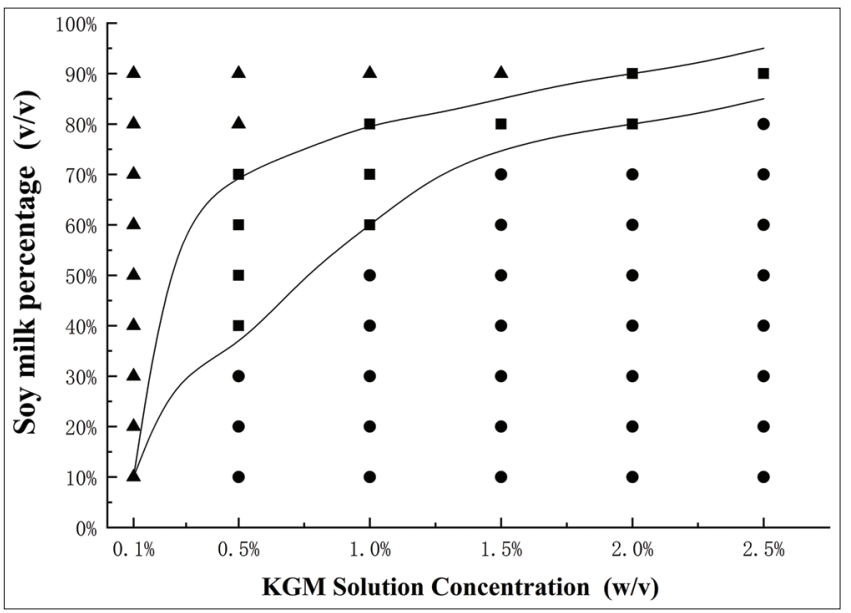

Fig 2. Phase separation diagram of KGM-soymilk mixture at $4^{\circ} \mathrm{C}$ for $12 \mathrm{~h}$ ( $\boldsymbol{\Delta}$, unstable zone; $\mathbf{n}$, metastable zone;, stable zone. Two solid lines are secant lines for three zones).

\section{Viscosity analysis}

Adding KGM into food could not only change the texture of food, but also improved the rheological properties of foods due to the colloidal stability of KGM (Devi et al., 2017). The stability of KGM-soy milk mixture was investigated by measuring the viscosity of the mixture system. Fig. 3 shows the influence of KGM concentration on the viscosity of the mixed system in case of the volume percentage of soy milk was fixed. It could be seen from the experimental results that the range of viscosity of the mixed solution with different KGM concentration was large.

As shown in Fig. 3, the viscosity of the mixed solutions increased with the increase of KGM concentration. In general, polysaccharides have high viscosity, therefore, the viscosity of mixture system could be increased. Moreover, the water retention of the emulsion could be improved by the addition of polysaccharides, and that avoided the evaporation or loss of water during short time, and then improved the stability of the mixture(Onsaard et al., 2014; Le et al., 2017). Liu et al(Liu et al., 2013) added KGM to egg white protein gel and also found that the water retention of protein-polysaccharide system could be improved, which might be attributed to the high viscosity of KGM in water and high ability of trapped water within the blending system. From the phase separation diagram of Fig. 2, we could also find that, as the concentration of KGM increased, the mixed system was more stable due to the high viscosity of the mixture. In other words, soy proteins were more firmly fixed by the colloid network of KGM under high viscosity, and they were dispersed evenly, which would be confirmed in the following CLSM.

\section{Turbidity analysis}

Fig. 4 shows the turbidity of the KGM-soy milk mixture system. The results indicated that the turbidity values of the mixture containing different KGM

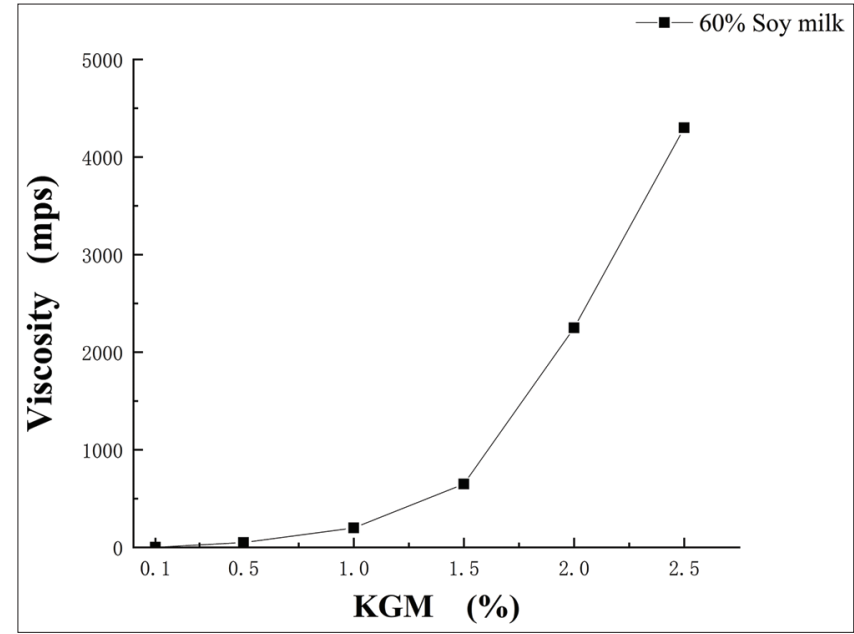

Fig 3. Viscosity of the KGM-soy milk mixture at room temperature. 


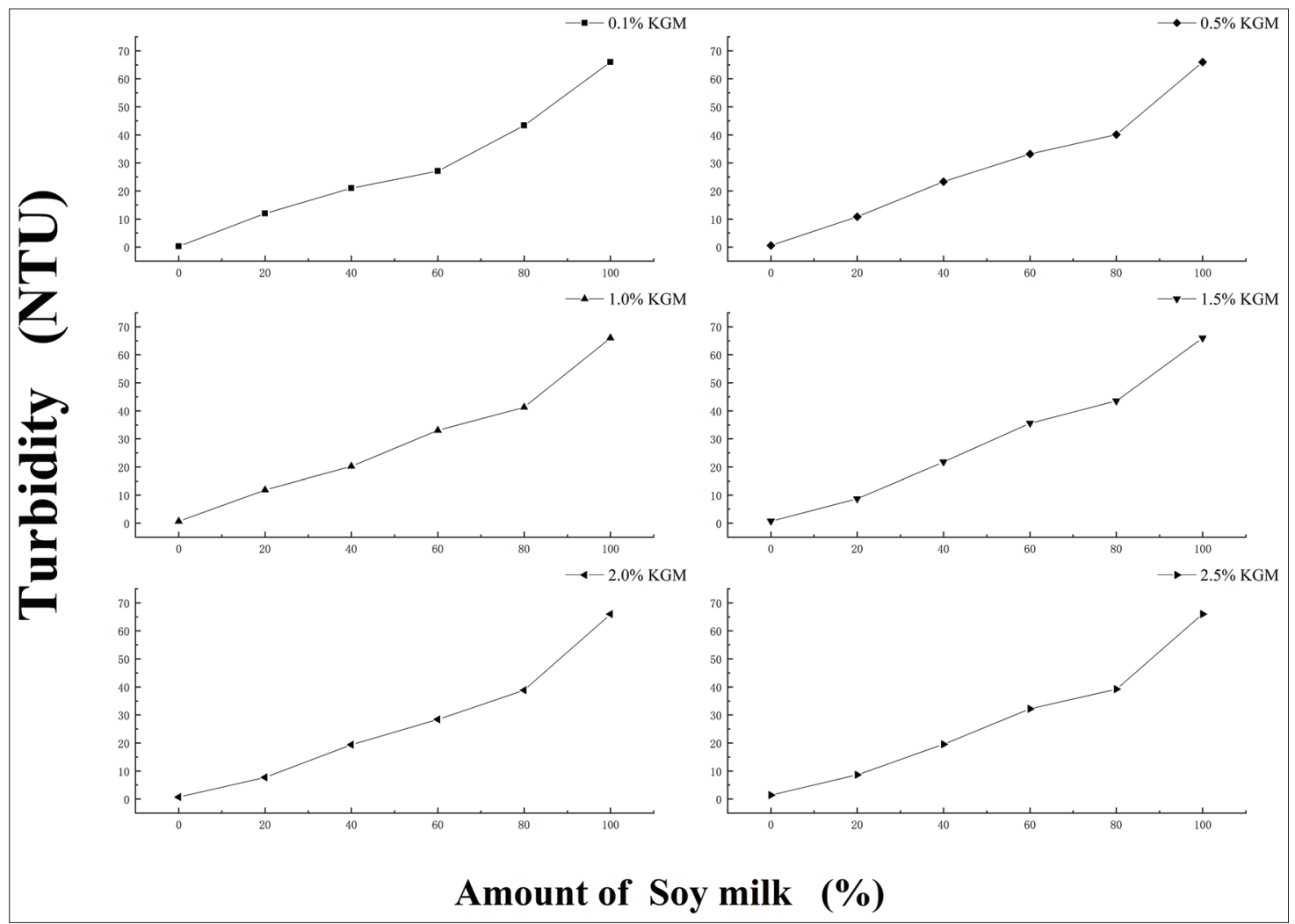

Fig 4. Turbidity of KGM- soy milk mixture.

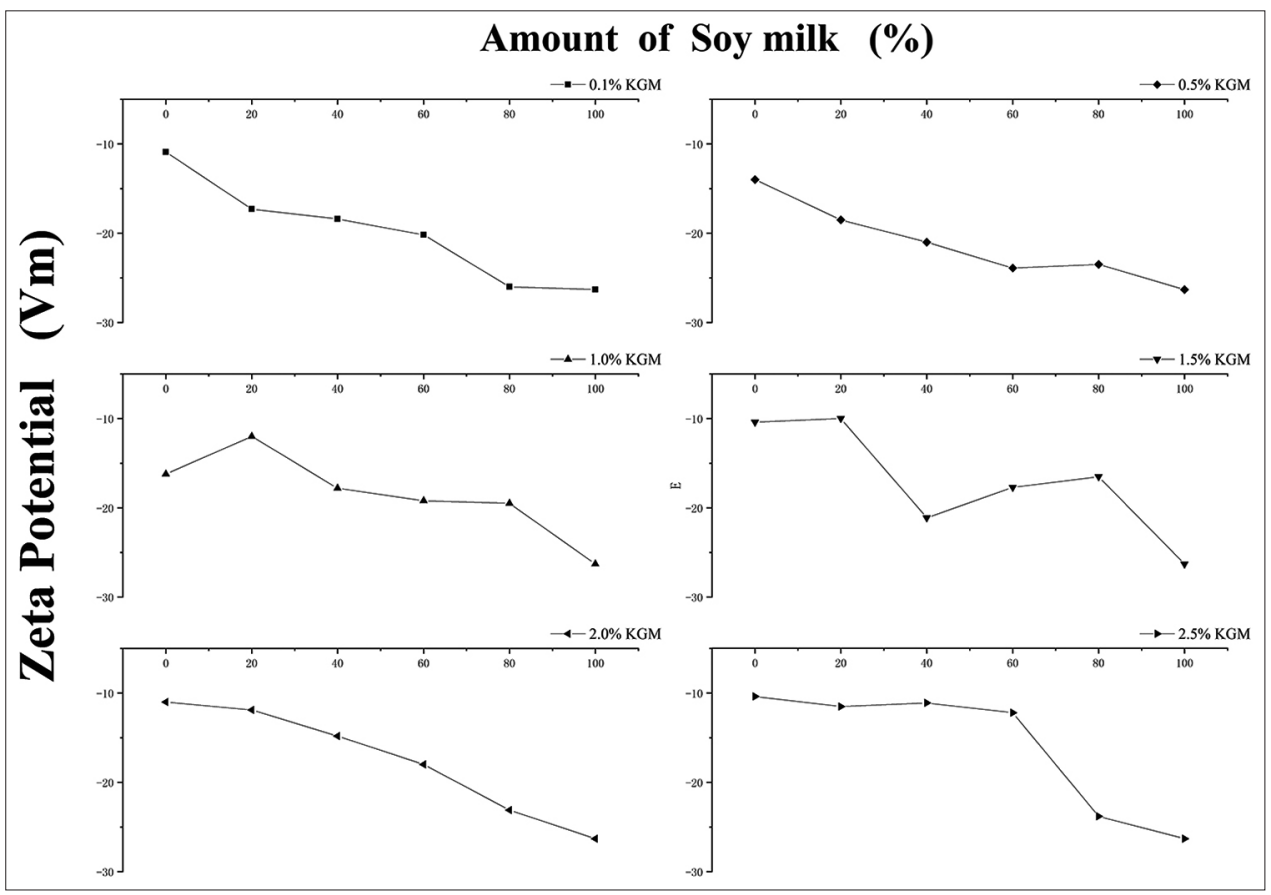

Fig 5. Zeta potential of the KGM-soy milk mixture.

concentrations $(0.1-2.5 \%)$ were all around $1 \mathrm{NTU}$ without significant difference. Under the same volume percentage of soy milk, the turbidity of the mixture system was higher than that of the pure KGM solution, which indicating that soy milk plays a key role in the turbidity of the mixture. The turbidity of the mixture showed a correlation with the ratio of protein and polysaccharide. Generally, turbidity was mainly derived from the altering in mass and size for the aggregates in the solution, and then the altering in turbidity was considered to be the outcome 


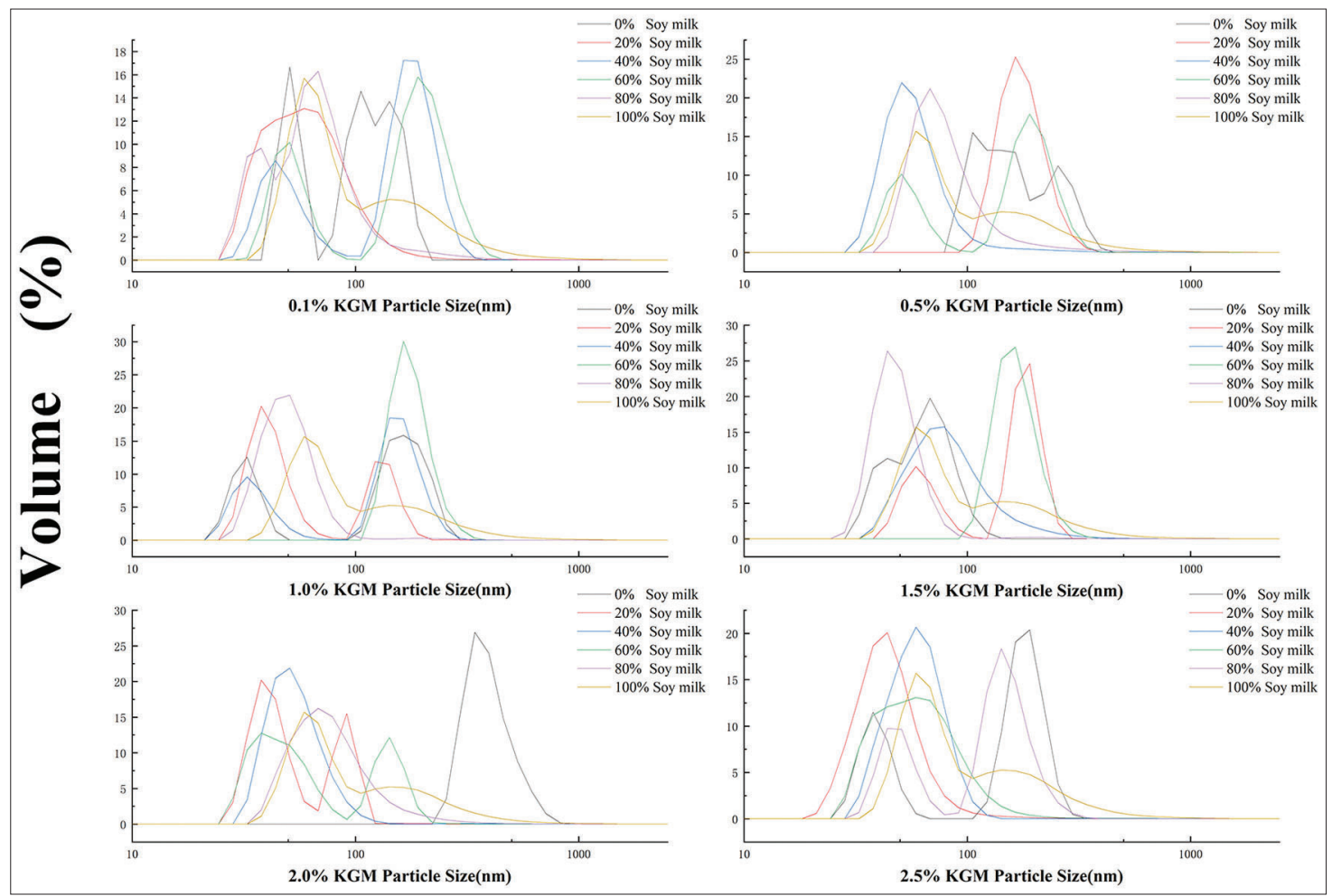

Fig 6. Particle size distribution of the KGM-soy milk mixture.

of the formation and decomposition of the protein/ polysaccharide coagulates(Ru et al., 2012). Therefore, the formation and decomposition of aggregates in protein/ polysaccharide mixtures could be comprehended by the change in the value of turbidity(Xia et al., 1993). The finding of Turgeon and co-workers also demonstrated that the significant increase in turbidity was due to the aggregation of mixtures(Turgeon et al., 2003). Under the same volume percentage of soy milk and the low KGM concentration, the effect of KGM concentration on the turbidity of the mixture was little. The turbidity of the mixture with high KGM concentration was generally lower than that of the one with lower KGM. This phenomenon might be explained that KGM can improve the dispersion of the aggregated protein particles and it also proved that KGM with high viscosity could stabilize protein granules.

\section{Potential analysis}

Zeta potential is the potential difference between a dispersed medium and a fixed liquid layer attached to a dispersed droplet, which is related to the stability of emulsions (Wang et al., 2011). In general, emulsion with high absolute Zeta potential is relatively stable for electrostatic interaction, whereas emulsion with low absolute Zeta potential is relatively unstable for trend in coagulation or flocculation (Wang et al., 2010). Fig. 5 shows the Zeta potential of the KGM-soy milk mixture. As shown in Fig. 5, when the KGM concentration was fixed, the Zeta potential of the mixture with a higher volume percentage of soy milk was higher,

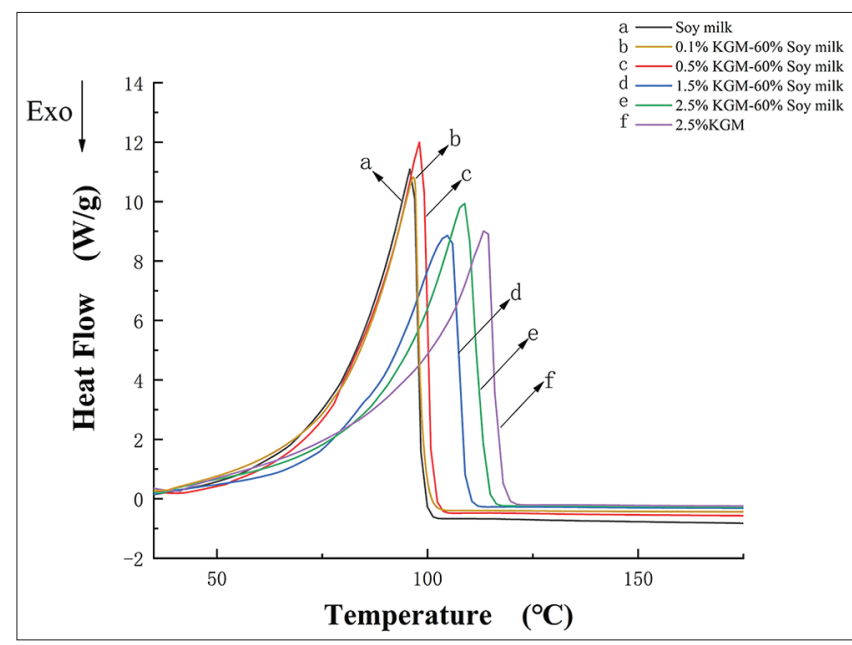

Fig 7. DSC curve of soy milk, KGM and KGM- soy milk mixture.

which indicated it seems to be more stable. However, the results of the phase separation diagram were just the opposite. It may be explained that when the proportion of the soy milk was high, KGM was not enough to cover the protein particles completely, and then, causing the aggregation and settlement of the protein particles.

The experimental results indicated that at the same concentration of KGM (including the lower concentration of KGM), the Zeta potential of the mixed system with a higher percentage of soy milk was higher and the mixed system should be more stable. But the phase diagram showed that, 

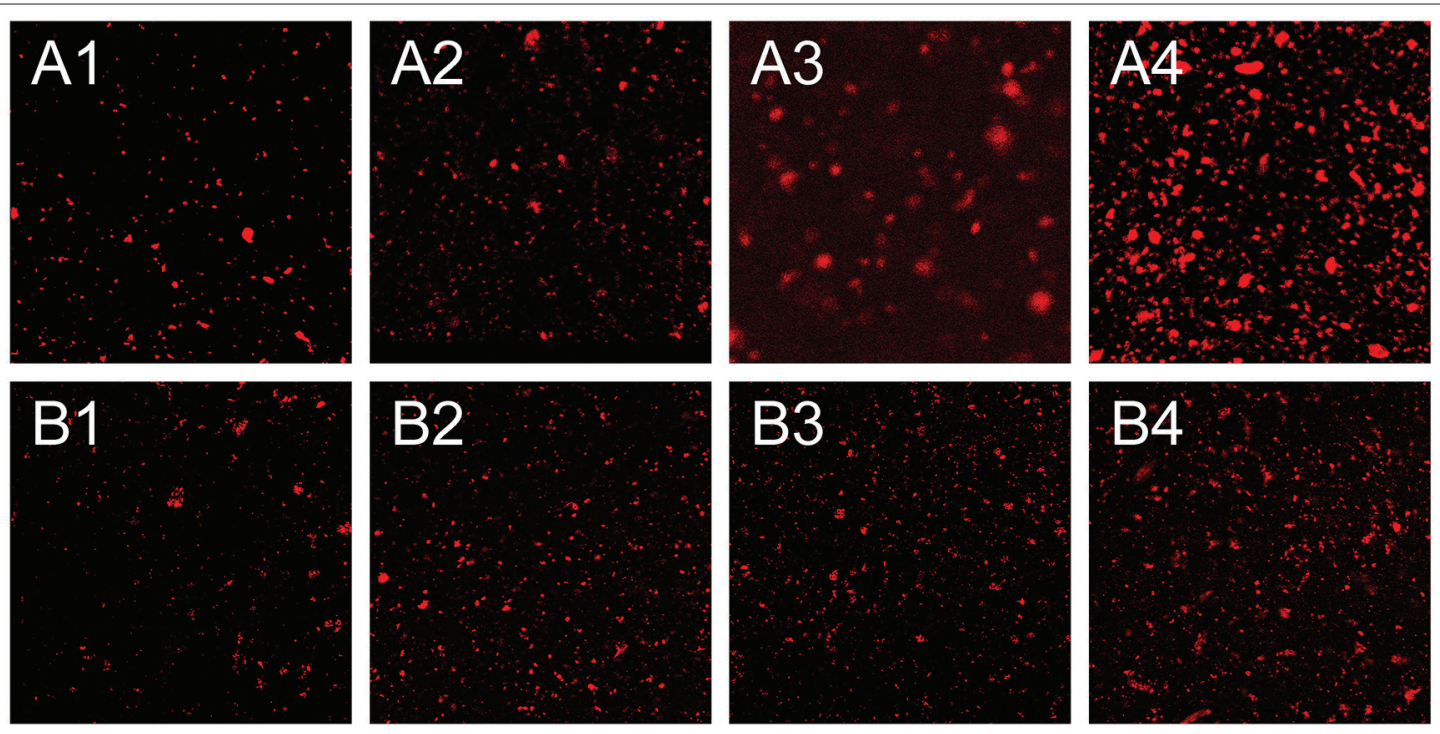

Fig 8. CLSM images of the KGM-soy milk (A1: 0.5\% KGM-20\% soy milk; A2: 0.5\% KGM-40\% soy milk; A3: 0.5\% KGM-60\% soy milk; A4: 0.5\% KGM-80\% soy milk; B1: $2.5 \%$ KGM-20\% soy milk; B2: $2.5 \%$ KGM- $40 \%$ soy milk; B3: $2.5 \%$ KGM-60\% soy milk; B4: $2.5 \%$ KGM-80\% soy milk).

when KGM concentration was low, the mixed system with a high volume percentage of soy milk might appear to phase separation, which could be explained that at a high proportion of the soy milk, the quantity of KGM was not enough to completely cover the proteins, then led to the aggregation and sedimentation. And this kind of effect was greater than the electrostatic repulsion between macromolecules.

\section{Particle size distribution analysis}

Fig. 6 shows the particle size distribution of the KGM-soy milk mixture. The range of particle size was from $10 \mathrm{~nm}$ to $1000 \mathrm{~nm}$. The aggregation of particles resulted in a wide range of the particle size distribution. The range of the particle size distribution of the mixture with low KGM concentration was wide. And, with the increase of KGM concentration, the diagram of the particle size distribution showed relatively close double peaks, indicating that the particle size distribution was influenced by the KGM concentration in the mixture.

Hemar et al.(Hemar et al., 2001) found that xanthan gum could promote protein toformm larger aggregates in the whey-xanthan gum mixture. The lyophilic particles became deformed at the oil-water interface. $\mathrm{Ni}$ and co-workers found in the KGM-zein mixture system that with the increase of zein content, the particles in the mixture system gradually increased and the uniformity decreased (Ni et al., 2018). Usually, latex particles were more easily deformed at the interface for their softness and porosity, and the emulsion stability was affected by the combination of particles deformability and surface viscoelasticity (Pinaud et al., 2014). Semenova drew a similar conclusion in his review on the associative interactions of polysaccharide-protein (Semenova, 2017). In the mixed system with low concentration of KGM, KGM molecules were insufficient to completely cover proteins and might lead to the aggregation of proteins with their neighbors. However, in the mixed system with high concentration of KGM, KGM also increased the viscosity of the mixing system, which could limit the movement of droplets and prevent particles from aggregating(Jafari et al., 2008).

\section{Differential scanning calorimetry}

The thermal behavior of KGM- soy milk mixture system was analyzed by DSC. It was generally accepted that the structural and functional properties of proteins could be influenced by thermal behavior. The hydrogen bond, disulfide bond, electrostatic interaction and hydrophobicity of proteins could be destroyed by heat treatment, resulting in the denaturation of proteins(Liu et al., 2012). Fig. 7 shows the DSC curves of soy milk, KGM solution, and KGMsoy milk mixture (fixed at $60 \%$ volume percentage) with $0.1 \%, 0.5 \%, 1.5 \%$ and $2.5 \%(\mathrm{w} / \mathrm{v}) \mathrm{KGM}$ concentration, respectively. As shown in Fig. 7, the highest endothermic peak temperature of soy milk was the lowest, and that of KGM was the highest. The highest endothermic peak temperature of soybean milk was increased by adding KGM, which increased with the increase of KGM concentration.

As the finding of Zhu(Zhu et al., 2009), when the concentration of guar gum increased from $0.05 \%$ to $0.5 \%$, the endothermic peak of soy globulin moved to high temperature, and the thermal and functional properties of soy globulin were improved by the incorporation of guar gum. Usually, the plant protein in soy milk would cause coagulation of protein molecules under the action of heat. However, the attached KGM could inhibit the proteins to aggregate with their neighbors, which then changed the thermal properties of the soy milk and 
improved the stability of the soy milk (Begum et al., 2003). Ibanoglu(Ibanoglu, 2005) had reported the influence of hydrocolloids(pectin, guar gum, $i$-carrageenan) on the thermal denaturation of proteins and found similar results.

\section{Microstructure of the KGM-soy milk mixture}

Fig. 8 shows the CLSM image of the KGM-soy milk, where the protein part labeled with Nile red was in the red region and the part of KGM was in the dark region. As it is shown in Fig. 8, the proteins in the mixture a relatively evenly distributed at the macroscopic level. As the percentage of soybean milk increased, the density of protein particles increased significantly. This change trend was similar to that reported by $\mathrm{Li}$ et al(Li et al., 2008) on the KGM-milk mixture. For group A(at low KGM concentrations), with the increase of soy milk, the agglomeration of proteins was obvious and especially was the most serious at $80 \%(\mathrm{v} / \mathrm{v})$ of soy milk. It might be caused by the low viscosity of KGM, and the colloidal network formed by KGM was insufficient to cover the protein particles, which easily result in the phase separation.

For group B (at high KGM concentrations), no matter how the soybean milk percentage changed, the distribution of soy milk granules was very uniform in the mixed system. It could be explained as follows: The colloid network formed by enough polysaccharide molecules served as a spaced space between protein particles. On the one hand, it could immobilize the protein particles, which delayed the phase separation and the emulsion stratification caused by gravity. On the other hand, it could prevent protein particles from aggregating by locking the hydrophobic binding site on the surface(Ibanoglu, 2005). In other words, the mixture was relatively stable at high KGM concentration.

\section{CONCLUSIONS}

In this work, the KGM-soy milk complex was prepared by adding KGM in soy milk. The miscibility and stability of the KGM-soy milk mixture depended on the delicate balance between the amount of KGM and that of soy milk in the system. The results showed that the KGM-soy milk mixture could form three system, i.e., unstable, metastable and stable system. Under the proper percentage soy milk, with the increase of KGM concentration, the mixture system was more stable. The study of the stability, phase behavior, thermal property and microstructure of KGMsoy milk mixture might provide a feasible solution for the development of novel compound plant beverage.

\section{ACKNOWLEDGMENTS}

This work was supported by the National Natural Science Foundation of China(No: 51263009), the Project of Team
Research for Excellent Mid-Aged and Young Teachers of Higher Education of Hubei Province (No: T201006).

\section{CONFLICTS OF INTEREST}

The authors declare that there is no conflict of interest.

\section{Authors' contributions}

Dating Tian conceived and designed the experiments, analyzed and interpreted data, drafted the article: Kai An performed the experiments and analyzed the data: Huiting Kang helped to perform the experiments and the analysis. All authors read and approved the final manuscript.

\section{REFERENCES}

Abbasi, S. and S. Mohammadi. 2013. Stabilization of milk-orange juice mixture using persian gum: Efficiency and mechanism. Food Biosci. 2: 53-60.

Ahmed, E., R. P. Rita, S. Huq, E. Kabir, M. S. Rana and M. A. R. Mazumder. 2019. Development of functional condensed milk from coconut milk and soy milk. Asian Food Sci. J. 6(4): 1-7.

Begum, S., A. Saito, X. Xu and A. Kato. 2003. Improved functional properties of the ovoinhibitor by conjugating with galactomannan. Biosci. Biotechnol. Biochem. 67(9): 1897-1902.

Behera, S. S. and R. C. Ray. 2016. Nutritional and potential health benefits of konjac glucomannan, a promising polysaccharide of elephant foot yam, Amorphophallus konjac K. Koch: A review. Food Rev. Int. 33(1): 22-43.

Chun, J. Y., G. P. Hong, S. Surassmo, J. Weiss, S. G. Min and M. J. Choi. 2014. Study of the phase separation behaviour of native or preheated wpi with polysaccharides. Polymer. 55(16): 4379-4384.

Dai, S., F. Jiang, N. P. Shah and H. Corke. 2017. Stability and phase behavior of konjac glucomannan-milk systems. Food Hydrocoll. 73: $30-40$.

Dalgleish, D. G. 2006. Food emulsions-their structures and structureforming properties. Food Hydrocoll. 20(4): 415-422.

de Kruif, C. G. and R. Tuinier. 2001. Polysaccharide protein interactions. Food Hydrocoll. 15(4): 555-563.

Devi, N., M. Sarmah, B. Khatun and T. K. Maji. 2017. Encapsulation of active ingredients in polysaccharide-protein complex coacervates. Adv. Colloid Interface Sci. 239: 136-145.

Giri, S. K. and S. Mangaraj. 2012. Processing influences on composition and quality attributes of soymilk and its powder. Food Eng. Rev. 4(3): 149-164.

Hemar, Y., M. Tamehana, P. A. Munro and H. Singh. 2001. Viscosity, microstructure and phase behavior of aqueous mixtures of commercial milk protein products and xanthan gum. Food Hydrocoll. 15(4): 565-574.

Hussein, M. M., F. A. M. Hassan, H. H. A. Daym, A. Salama, A. K. Enab and A. A. Abd El-Galil. 2011. Utilization of some plant polysaccharides for improving yoghurt consistency. Ann. Agric. Sci. 56(2): 97-103.

Ibanoglu, E. 2005. Effect of hydrocolloids on the thermal denaturation of proteins. Food Chem. 90(4): 621-626.

Jafari, S. M., E. Assadpoor, Y. He and B. Bhandari. 2008. Recoalescence of emulsion droplets during high-energy 
emulsification. Food Hydrocoll. 22(7): 1191-1202.

Kontogiorgos, V., S. M. Tosh and P. J. Wood. 2009. Phase behaviour of high molecular weight oat $\beta$-glucan/whey protein isolate binary mixtures. Food Hydrocoll. 23(3): 949-956.

Laurent, M. A. and P. Boulenguer. 2003. Stabilization mechanism of acid dairy drinks (add) induced by pectin. Food Hydrocoll. 17(4): 445-454.

Le, X. T., L. E. Rioux and S. L. Turgeon. 2017. Formation and functional properties of protein-polysaccharide electrostatic hydrogels in comparison to protein or polysaccharide hydrogels. Adv. Colloid Interface Sci. 239: 127-135.

Li, X., F. Deng, Y. Hua, A. Quu, C. Yang and S. Cui. 2008. Effect of molecular weight of dextran on the phase behavior and microstructure of preheated soy protein/dextran mixtures. Carbohydr. Polym. 72(1): 160-168.

Liu, J., K. Zhu, T. Ye, S. Wan, Y. Wang, D. Wang, B. Li and C. Wang. 2013. Influence of konjac glucomannan on gelling properties and water state in egg white protein gel. Food Res. Int. 51(2): 437-443.

Liu, Y., G. Zhao, M. Zhao, J. Ren and B. Yang. 2012. Improvement of functional properties of peanut protein isolate by conjugation with dextran through maillard reaction. Food Chem. 131(3): 901-906.

Ni, X., K. Wang, K. Wu, H. Corke, K. Nishinari and F. Jiang. 2018. Stability, microstructure and rheological behavior of konjac glucomannan-zein mixed systems. Carbohydr. Polym. 188: $260-267$.

Omoni, A. O. and R. E. Aluko. 2005. Soybean foods and their benefits: Potential mechanisms of action. Nutr. Rev. 63(8): 272-283.

Onsaard, E., J. Putthanimon, J. Singthong and P. Thammarutwasik. 2014. Influence of maltodextrin and environmental stresses on stability of whey protein concentrate/kappa-carrageenan stabilized sesame oil-in-water emulsions. Food Sci. Technol. Int. 20(8): 617-628.

Pinaud, F., K. Geisel, P. Masse, B. Catargi, L. Isa, W. Richtering, V. Ravaine and V. Schmitt. 2014. Adsorption of microgels at an oilwater interface: Correlation between packing and $2 \mathrm{~d}$ elasticity. Soft Matter. 10(36): 6963-6974.

Pirestani, S., A. Nasirpour, J. Keramat, S. Desobry and J. Jasniewski. 2018. Structural properties of canola protein isolate-gum arabic maillard conjugate in an aqueous model system. Food Hydrocoll. 79: $228-234$.
Ru, Q., Y. Wang, J. Lee, Y. Ding and Q. Huang. 2012. Turbidity and rheological properties of bovine serum albumin/pectin coacervates: Effect of salt concentration and initial protein/ polysaccharide ratio. Carbohydr. Polym. 88(3): 838-846.

Semenova, M. 2017. Protein-polysaccharide associative interactions in the design of tailor-made colloidal particles. Curr. Opin. Colloid Interface Sci. 28: 15-21.

Sharafbafi, N., S. M. Tosh, M. Alexander and M. Corredig. 2014. Phase behaviour, rheological properties, and microstructure of oat $\beta$-glucan-milk mixtures. Food Hydrocoll. 41: 274-280.

Turgeon, S. L., M. Beaulieu, C. Schmitt and C. Sanchez. 2003. Protein-polysaccharide interactions: Phase-ordering kinetics, thermodynamic and structural aspects. Curr. Opin. Colloid Interface Sci. 8(4-5): 401-414.

Wang, Y., D. Li, L. J. Wang and B. Adhikari. 2011. The effect of addition of flaxseed gum on the emulsion properties of soybean protein isolate (spi). J. Food Eng. 104(1): 56-62.

Wang, Y., D. Li, L. J. Wang, S. J. Li and B. Adhikari. 2010. Effects of drying methods on the functional properties of flaxseed gum powders. Carbohydr. Polym. 81(1): 128-133.

Xia, J., P. L. Dubin and H. Dautzenberg. 1993. Light scattering, electrophoresis, and turbidimetry studies of bovine serum albumin-poly(dimethyldiallylammonium chloride) complex. Langmuir. 9(8): 2015-2019.

Xiong, W., C. Ren, M. Tian, X. Yang, J. Li and B. Li. 2018. Emulsion stability and dilatational viscoelasticity of ovalbumin/ chitosan complexes at the oil-in-water interface. Food Chem. 252: $181-188$

Ye, R. and F. Harte. 2014. High pressure homogenization to improve the stability of casein-hydroxypropyl cellulose aqueous systems. Food Hydrocoll. 35: 670-677.

Yuliarti, O., K. H. Mei, Z. K. X. Ting and K. Y. Yi. 2019. Influence of combination carboxymethylcellulose and pectin on the stability of acidified milk drinks. Food Hydrocoll. 89: 216-223.

Zhao, G. Y., H. J. Diao and W. Zong. 2013. Nature of pectin-proteincatechin interactions in model systems: Pectin-protein-catechin interactions. Food Sci. Technol. Int. 19(2): 153-165.

Zhu, J. H., X. Q. Yang, I. Ahmad, Y. Jiang, X. Y. Wang and L. Y. Wu. 2009. Effect of guar gum on the rheological, thermal and textural properties of soybean $\beta$-conglycinin gel. Int. J. Food Sci. Technol. 44(7): 1314-1322. 